\title{
Study on Effective Transmission Scheme Based on Fountain Codes in Wireless Multimedia Sensor Network
}

\author{
Ben-Shun Yi, Wei-Qing Yao, Wei-Zhong Li, Kang Qiu, Tai-Qi Huang \\ Shenzhen Institute of Wuhan University \\ School of Electronic Information, Wuhan University \\ E-mail: yibs@whu.edu.cn
}

\begin{abstract}
Wireless Multimedia Sensor Network (WMSN) achieves the multimedia information from the distributed sensor nodes. In this paper, we design a transmission scheme to ensure the efficient and reliable data transmission in WMSN. Firstly, LT codes are employed as the channel coding. A method is proposed to optimize the degree distribution for LT codes based on Binary Exponential Distribution (BED) and Bird Swarm Algorithm (BSA) to improve both the decoding efficiency and successful decoding rate. Moreover, aiming at the spatial and temporal redundancy of the node information, we try to study the cooperative information processing method with distributed compressed sensing and image fusion to improve the efficiency of data transmission and the quality of the images. Simulation results show that the proposed transmission scheme can solve the bottleneck problem of the efficient and reliable transmission in the WMSN.
\end{abstract} sensing

Keywords- WMSN; LT codes; image fusion; compressive

\section{INTRODUCTION}

Wireless Multimedia Sensor Network (WMSN) is composed of multimedia sensor nodes capable of wireless communication and information processing. These nodes distribute throughout the space, collaboratively perceive multimedia information of the external environment, and transfer to end users. In recent years, the explosion of wireless mobile intelligent terminal promotes the rapid development of the WMSN, brings a broad application prospect particularly in security monitoring, environmental monitoring, intelligent household, health care, etc. Multimedia information has the characteristics of real-time, large data and QoS-aware of WMSN, which makes great challenge for WMSN to realize high and reliable data transmission in complex wireless network environment while the nodes' energy consumption, bandwidth are limited.

Due to the strong time and spatial correlation between sampling images from the nodes, the information from multiple nodes can be fused to eliminate redundant information so as to further reduce network traffic. And the image quality also can be improved through image fusion techniques. In 2013, the method for adaptive fusion of multimodal surveillance images based on Non-Subsampled Contourlet Transform (NSCT) was proposed by D Egfin
Nirmala, which uses Compression Sensing (CS) technology to reduce redundant information so as to decrease energy consumption and bandwidth [1]. In 2014, an information fusion mechanism based on granularity of information was proposed by $\mathrm{Z}$ J Zhang, which greatly reduces the transmission data volume and energy consumption [2]. In 2015, a multi-component image fusion method based on Morphological Component Analysis (MCA) in WMSN was proposed, which reduces the amount of image transmission by effectively decomposing the visible image into smooth and texture components [3]. However, most of the methods only consider spatial correlation. And the high algorithm complexity is also a fatal disadvantage.

Fountain codes can be more suitable for application in WMSN compared to traditional channel coding technique, mainly because it has many outstanding characteristics such as it is not sensitive to packet loss and the order of received packets. It has almost ideal receiving efficiency which is adapted to the time-varying channel and doesn't need feedback. However, traditional fountain code needs to transfer a large number of redundant coded packets to ensure success of receiver's decoding, which may results in low transmission efficiency and high storage pressure to convergence node. Therefore, how to reduce decoding packets to enhance WMSN's transmission efficiency turns to be a key problem to be considered before introducing fountain codes to ensure reliable transmission of multimedia streaming data. In 2014, LT codes was used in WSN by Anuj and the idea of adapting LT codes for Forward Error Correction (FEC) by the application of BPSK erasure zone is proposed, which gets better transmission reliability compared to $\mathrm{BCH}$ code under the same conditions [4]. In 2015, a reliable data transfer protocol for WSN based on fountain codes was proposed by Arghavan and successfully reduced the number of data packets that source node needs to transfer [5]. In 2015, a scheme for multimedia data stream transmission in WMSN based on Unequal Error Protection (UEP) fountain codes were proposed by Jofina Jose and significantly enhanced transmission efficiency [6].But the scheme failed to reach optimal performance for it didn't optimize the coding strategy according to decoding information.

In this paper, we devote to design an effective transmission scheme for the multimedia stream in WMSN. 
The contributions of this work are mainly summarized as follows:1) We use LT code as channel coding technique in WMSN and propose a method to improve the efficiency of information transmission by optimizing the degree distribution for LT codes.2) We propose a novel method based on image fusion and compressive sensing to eliminate redundant information.

The remainder of the paper is organized as follows. Section 2 describes the structure of WMSN and the information transmission flow. A method to optimize the degree distribution for LT codes is proposed in Section 3. A novel approach based on image fusion and compressive sensing is presented in Section 4. Section 5 concludes the paper.

\section{NETWORK MODEL}

The structure of WMSN is shown in Fig.1. According to the resource allocation and dealing ability, the nodes are divided into sensor nodes, fusion nodes and aggregation nodes at the terminal to construct the cluster structure as Fig.1(a). And the principle is that the operation burden of the mass resource-constrained sensor nodes at the front-end is transferred to the few fusion nodes or the aggregation nodes whose dealing ability and storage resource are improved. Taking the image information for example, the workflow is presented in Fig.1 (b).

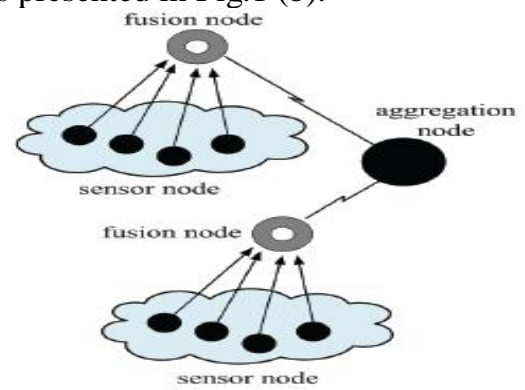

(a) topology structure

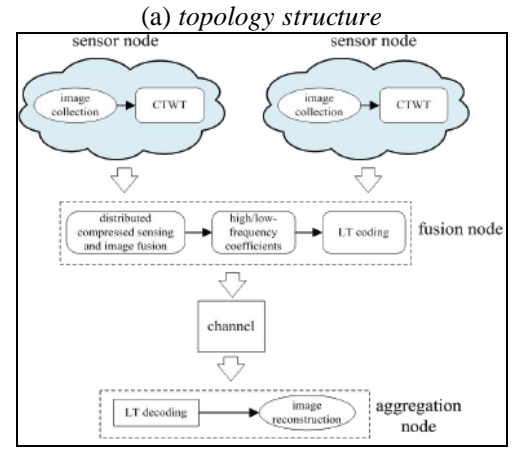

(b) workflow

Figure 1. Model of WMSN.

\section{MULTIMEDIA INFORMATION TRANSMITTING}

As the channel coding, LT codes are applied to transmit the multimedia information from the fusion node to the aggregation node in the WMSN. The degree distribution greatly influences the performance of LT codes [7]. In order to obtain a better degree distribution, a method is proposed to optimize the degree distribution for LT codes based on Binary Exponential Distribution (BED) [8] and Bird Swarm Algorithm (BSA) [9].

\section{A. Degree Distribution Optimization Based on BSA}

The decoding performance of BED outperforms RSD when the overhead is small, and its successful decoding rate reaches more than $80 \%$. The successful decoding rate for LT codes [7] rises quickly as the overhead increases, while BED almost keeps unchanged. In order to combine the advantages of BED and RSD, we use BSA [9] to search the optimal degree distribution for LT codes between these two. BSA can be efficiently extracted from the bird swarms to optimize problems by imitating foraging behavior, vigilance behavior and flight behavior of the birds.

The key factors of the proposed algorithm can be described as follows:

1) Initial bird swarms:

The bird corresponds to the degree distribution. The initial bird swarms are generated based on RSD and BED. Assume RSD is defined as $\mu(\cdot)$ and BED is defined as $b(\cdot) \quad$. Each bird has $\mathrm{k}$ parameters $\boldsymbol{\Omega}=(\Omega(1), \Omega(2), \cdots, \Omega(k))$, where $\Omega(i) \in[b(i), \mu(i)]$ $i=1,2,3, \cdots, k$. We obtain $\mathrm{m}$ initial bird swarms $\left\{\boldsymbol{\Omega}_{1}, \boldsymbol{\Omega}_{2}, \cdots, \boldsymbol{\Omega}_{m}\right\}$ randomly.

2) Objective function:

The expected ripple size is employed to present the objective function for BSA. The ripple is defined as the set of degree-1 encoding symbols in the decoding process of LT codes, expressing the relationship between successful decoding rate and the degree of encoding symbols in a certain extent [7]. The expected ripple size is given as [10]:

$$
R_{\Omega}(n)=(1+\varepsilon)(k-n)\left(\Omega^{\prime}\left(\frac{n}{k}\right)+\frac{1}{1+\varepsilon} \ln \frac{k-n}{k}\right)+O(1)
$$

where $\mathrm{k}$ is the number of input numbers, $\mathrm{n}$ is the number of decoding input symbols, $\Omega(\cdot)$ is the degree distribution, $\varepsilon=(N-k) / k$ is defined as the overhead, and $N=(1+\varepsilon) \cdot k$ is the number of encoding symbols have been received for successful decoding.

In order to comply with the properties of a good degree distribution in terms of the expected ripple size [11], the ripple size should both keep large enough during the decoding process and reduce the variation as the entry and removal of encoding symbols, so we consider increasing the mean of expected ripple size and decreasing its variation simultaneously. Our objective is to maximize 


$$
\vartheta=\frac{1}{k} \sum_{n=0}^{k-1} R_{\Omega}(n)-\alpha \cdot \frac{1}{k} \sum_{n=0}^{k-1}\left(R_{\Omega}(n)-\frac{1}{k} \sum_{n=0}^{k-1} R_{\Omega}(n)\right)^{2}
$$

where $\alpha \in(0,1)$

After evaluating and iterating repeatedly, we can achieve the optimal degree distribution.

\section{B. Simulation Results}

We verify the effectiveness of the optimization method (hereafter called LT-BSA) by 1000 encoding/decoding simulations with parameters $c=0.1, \delta=0.005$.

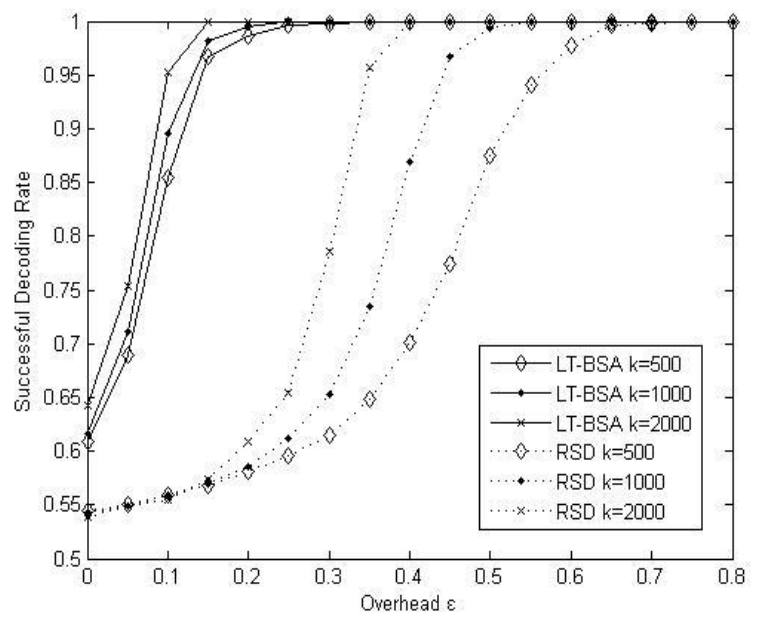

Figure 2. Successful decoding rate versus overhead with different k.

The successful decoding rate versus the overhead for LT-BSA with parameter $\alpha=0.25$ and $\varepsilon=0.1$ is shown in Fig.2. Compared to RSD, the higher successful decoding rate is obtained by using LT-BSA. Table I shows the average overhead needed to decode all $\mathrm{k}$ input symbols, the average degree per encoding symbol, and the average consuming time per encoding/decoding process.

TABLE I. PERFORMANCE COMPARISON FOR FOR RSD AND LT-BSA

\begin{tabular}{c|ccrlll}
\hline $\begin{array}{l}\text { Input } \\
\text { symbols }\end{array}$ & \multicolumn{4}{|l}{ Avg. Overhead (\%)Avg. degree $\begin{array}{l}\text { Avg. Consuming Time } \\
(\mathrm{s})\end{array}$} \\
\hline$k$ & RSD & LT-BSA & RSD & LT-BSA RSD & LT-BSA \\
500 & 70.3 & 36.5 & 9.9 & 5.1 & 4.6 & 3.7 \\
1000 & 50.7 & 18.2 & 11.7 & 6.5 & 17.9 & 15.5 \\
2000 & 40.6 & 15.1 & 13.4 & 6.9 & 83.7 & 65.6 \\
\hline
\end{tabular}

Furthermore, in order to verify the effectiveness of the LT-BSA compared to the RSD, the monkey image (grayscale $256 \times 256 \times 8=524288$ bit ) is used as the transmission data. 524288 bit is divided into 1024 input symbols averagely. The original image is recovered forcibly when the decoder receives 1180 encoding symbols. In Fig.3, it is obvious that the recovered image is distorted seriously by using RSD, while the image can be recovered completely for LT-BSA.
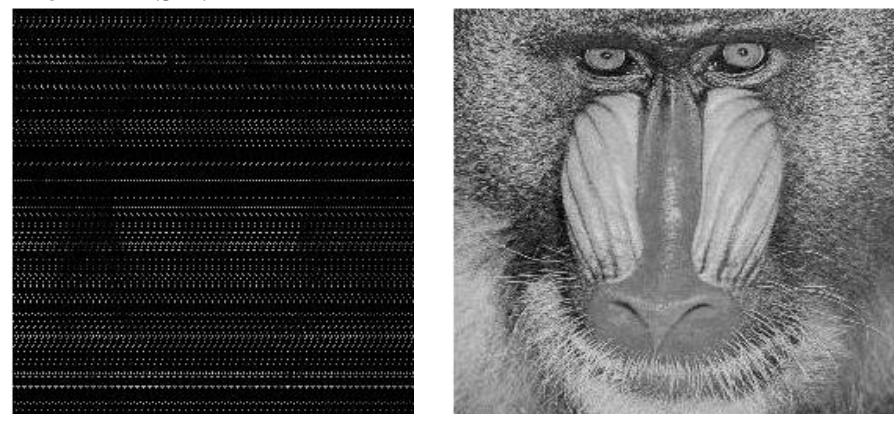

(a) Image transmission based on RSD

(b)Image transmission based on LT-BSA

\section{MULTIMEDIA INFORMATION PROCESSING}

In order to achieve efficient transmission of multimedia information, we process multimedia information based on image fusion and compressive sensing in fusion node. The multimedia signal correlation is divided into two categories: inter-signal correlation and intra-signal correlation [12]. When the adjacent sensor nodes collect information in the same scene, we use the image fusion method to reduce the redundant information according to inter-signal correlation. The algorithm is summarized as follows:

- Step 1: A low-frequency sub-band and multi-directional high-frequency sub-bands are obtained by using Contourlet-Wavelet (CTWT) transformation [13].

- Step 2: Calculating the absolute value of high frequency coefficients, the coefficients which absolute values are bigger than others are used as the coefficients of the fused image.

- Step 3: Calculating the energy value of the low-frequency coefficients in $7 \times 7$ neighborhood, the low-frequency coefficients which energy values are bigger than others are used as the coefficients of the fused image.

- $\quad$ Step 4: Fused images are obtained by using inverse CTWT transformation.

When different cameras capture partial segments of the same scene, the sink node need to fuse partial segments to acquire a complete image. According to the above fusion algorithm, the fused image of two adjacent sensor node is shown in Fig.4. The simulation results show that the redundant data in the same scene overlap portion has been reduced. 


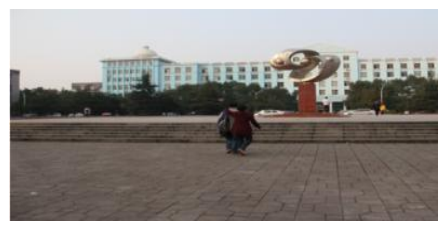

(a)The image of sensor node a

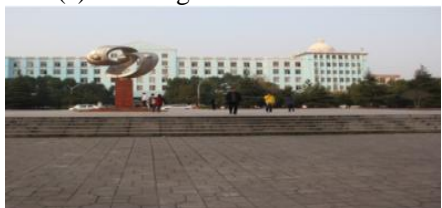

(b)The image of sensor node $b$

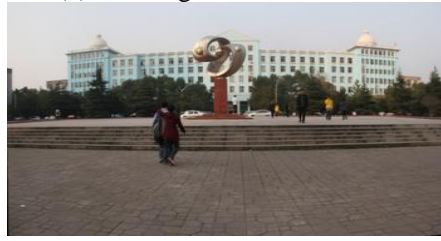

(c) Fused image of sink node

Figure 4. The result of image fusion.

When the sensor nodes collect information in different scenes, we use the compressive sensing to reduce the redundant information according to intra-signal correlation. Firstly, in the sensor node, we generate a random sample sequence and split it into multiple random sample sub-sequences; Then we use a random sample sub-sequences to sample the multimedia information and transmit the sampling information to the fusion node. Next, the compression signal is obtained by using observation matrix to reduce dimension random projection of sampled signals. Finally, the multimedia information is reconstructed in sink node using improved compressed samples matching algorithm [14].

\section{CONCLUSIONS}

In this paper, we study on a transmission scheme to improve the efficiency and reliability of multimedia information transmission in WMSN. LT code is used as the channel coding, and a method is proposed to enhance both the decoding efficiency and successful decoding rate by optimizing the degree distribution for LT codes. Furthermore, we propose a novel method based on image fusion and compressive sensing to eliminate redundant information in WMSN.

\section{ACKNOWLEDGEMENT}

This paper was supported by Basic Research Project of Shenzhen, China under Grant JCYJ20150630153917254, and National Natural Science Foundation of China under Grant 61371125.

\section{REFERENCES}

[1] Nirmala, D. Egfin, R. K. Vignesh, and V. Vaidehi. "Multimodal image fusion in visual sensor networks." Electronics, Computing and Communication Technologies (CONECCT), 2013 IEEE International Conference on. IEEE, 2013.

[2] Zhang, Zhen-Jiang, Chin-Feng Lai, and Han-Chieh Chao. "A green data transmission mechanism for wireless multimedia sensor networks using information fusion." Wireless Communications, IEEE 21.4 (2014): 14-19.

[3] Lin, Yih-Lon, Yu-Min Chiang, and Yi-Ling Tsai. "Morphological component analysis and least squares support vector machine for image super-resolution." Machine Learning and Cybernetics (ICMLC), 2015 International Conference on. Vol. 1. IEEE, 2015.

[4] Nayak, Amiya. "Performance analysis of LT codes and BCH codes in RF and FSO Wireless Sensor Networks." Advances in Computing, Communications and Informatics (ICACCI, 2014 International Conference on. IEEE, 2014.

[5] Modiri, Arghavan, and Shahram Yousefi. "Single-source two-terminal multicast networks with overlapping demands over the binary erasure channel." Information Theory (CWIT), 2015 IEEE 14th Canadian Workshop on. IEEE, 2015.

[6] Jose, Jofina, and S. M. Sameer. "A new unequal error protection technique for scalable video transmission over multimedia wireless networks." Signal Processing, Informatics, Communication and Energy Systems (SPICES), 2015 IEEE International Conference on. IEEE, 2015.

[7] Luby, Michael. "LT codes." null. IEEE, 2002.

[8] Al Agha, Khaldoun, Nour Kadi, and Ivan Stojmenovic. "Fountain Codes with XOR of Encoded Packets for Broadcasting and source independent backbone in Multi-hop Networks using Network Coding." Vehicular Technology Conference, 2009. VTC Spring 2009. IEEE 69th. IEEE, 2009

[9] Meng, Xian-Bing, et al. "A new bio-inspired optimisation algorithm: Bird Swarm Algorithm." Journal of Experimental \& Theoretical Artificial Intelligence (2015): 1-15.

[10] Karp, Richard, Michael Luby, and Amin Shokrollahi. "Finite length analysis of LT codes." Information Theory, 2004. ISIT 2004. Proceedings. International Symposium on. IEEE, 2004.

[11] Yen, Kuo-Kuang, et al. "Modified robust soliton distribution (MRSD) with improved ripple size for lt codes." Communications Letters, IEEE 17.5 (2013): 976-979.

[12] Baron, Dror, et al. "Distributed compressive sensing." arXiv preprint arXiv: 0901. 3403,2009

[13] Shu, Zhenghua, Guodong Liu, and Zhihua Xie. "An image coding of shape adaptive wavelet-based contourlet transform." Systems and Informatics (ICSAI), 2014 2nd International Conference on. IEEE, 2014.

[14] Chen, Chulong, and Michael D. Zoltowski. "A modified compressed sampling matching pursuit algorithm on redundant dictionary and its application to sparse channel estimation on OFDM." Signals, Systems and Computers (ASILOMAR), 2011 Conference Record of the Forty Fifth Asilomar Conference on. IEEE, 2011. 